\title{
The Incidence and Epidemiology of Tuberculosis in Sistan Region of Iran: An Update to the Past Researches
}

\author{
Reza Shirazinia ${ }^{1 *}$, Dariush Saadati ${ }^{2}$, Elyas Zeinali ${ }^{3}$, Abolfazl Panahi Mishkar ${ }^{4}$ \\ ${ }^{1}$ Faculty of Veterinary Medicine, University of Zabol, Zabol, Iran \\ ${ }^{2}$ Department of Food Hygiene and Quality Control, Faculty of Veterinary Medicine, University of Zabol, Zabol, Iran \\ ${ }^{3}$ Faculty of Veterinary Medicine, University of Zabol, Zabol, Iran \\ ${ }^{4}$ Human Ecology, Kerman University of Medical Sciences, Kerman, Iran
}

\section{*Correspondence to}

Reza Shirazinia,

Email: rezagalaxy47@gmail.com

Received July 17, 2017 Accepted October 6, 2017

Published online December 31, 2017

\begin{abstract}
Introduction: Tuberculosis (TB) is a chronic disease which spreads through respiration and develops lesions in lung or other organs. The most usual kind of TB is pulmonary TB. The highest incidence rate of TB is in the eastern neighbors of Iran rendering high TB incidence in Sistan and Baluchistan province of Iran. The current research was conducted to investigate the epidemiology of TB in the north of the province, the Sistan region.

Methods: In this survey, data of all suspected and established TB cases in the Zabol city was collected from their profiles in health care centers during 2010-2013. The trend of the disease, and its prevalence based on residency and gender were assessed.

Results: In this period of time, a total of 1800 TB cases were detected. Of these, $485(27 \%)$ were from urban areas and $1315(73 \%)$ were from rural areas. The trend of TB identification increased with a slope of nearly 46.4. In this research, the most resistance to drug was related to Isoniazid and Rifampin and the least resistance was to ethambutol and streptomycin.

Conclusion: Dust storms in this area because of successive droughts and other living conditions can make the region susceptible to TB disease. Therefore, people in this area, especially in rural regions, should be given sufficient education to be protected against this infection.

Keywords: Epidemiology, Sistan, Tuberculosis
\end{abstract}

Please cite this article as follows: Shirazinia R, Saadati D, Zeinali E, Panahi Mishkar A. The incidence and epidemiology of tuberculosis in Sistan region of Iran: an update to the past researches. Int J Basic Sci Med. 2017;2(4):189193. doi:10.15171/ ijbms.2017.35.

\section{Introduction}

Tuberculosis (TB) is a chronic infectious disease, that is often caused by Mycobacterium tuberculosis and sometimes by Mycobacterium bovis, Mycobacterium microti and Mycobacterium africanism. ${ }^{1}$ This disease spreads through respiration, food, and direct contact affecting lung or other parts of the body. The most common type of TB is pulmonary TB. This form of TB spreads by respiration of $M$. tuberculosis. Pulmonary TB is accounted for about $80 \%$ of TB cases with the most cases of adult infections, which are smear positive and highly contagious. ${ }^{2}$

Despite considerable progresses in diagnostic and therapeutic approaches, TB has continued to infect people from the past to the present. ${ }^{3}$ In the 19th century, this illness has been the most important cause of death worldwide..$^{4-6}$ Emergence of immunocompromised diseases like AIDS (HIV) has increased the TB incidence. ${ }^{1}$ Every year, 9 million people are infected with $\mathrm{TB}$, and 1.5-2 million die. This illness has been ranked as the 10th most important disease according to the global burden of the diseases. ${ }^{7}$ More than $90 \%$ of the TB related deaths happen in developing countries. Six countries with the most rates of infections in 2014 were India, Indonesia, China, Nigeria, Pakistan, and South Africa. ${ }^{8}$ Incidence rate of TB varies in different ages around the world. In poor countries, the most cases are developed among youths and teens; however, because of having more effective sanitary programs, this illness occurs in old and middle-aged

(C) 2017 The Author(s); Published by Zabol University of Medical Sciences. This is an open-access article distributed under the terms of the Creative Commons Attribution License (http://creativecommons.org/licenses/by/4.0), which permits unrestricted use, distribution, and reproduction in any medium, provided the original work is properly cited. 
individuals in developed countries. ${ }^{9}$

In developing countries, as high as $75 \%$ of incidences are among the most active economic ages (14-54 years old). Given that an adult with TB cannot work for about 3-4 months, hence $20 \%-30 \%$ of the revenue of the family is vanished and if a TB patient dies, the income of family for one year will be lost. ${ }^{7}$ Because of the importance of TB in Sistan region at the north of the Sistan and Baluchistan province of Iran, and due to the high incidence rate of TB in the eastern neighbors of the province, the current research was conducted to determine the incidence and epidemiology of TB in this endemic area.

\section{Methods}

In this survey, data of all suspected and established TB patients in Zabol city, the center of Sistan region, were collected during 2010-2013. The data was gathered from patients' profiles at the health care centers with contribution of TB center of Zabol city. Based on the localized health care rules, all physicians should report all suspected or established cases of TB to the sanitary center of the district and create profiles for all of them. Hence, this survey was conducted based on the reported information from the patients of TB that were collected by the sanitary centers and TB Center of Zabol city. Demographic information of the patients, type of TB, and information about drug resistance were collected. To evaluate the drug resistance, antibiogram test was carried out on 525 isolates of Mycobacterium and rapid test was performed for the ordinary antibiotics that were used in the first line of TB treatment (isoniazid, rifampin, ethambutol, and streptomycin). The incidence rate in this period was reported as incidence rate per 100000 of the population.

\section{Results}

During 2010-2013, 21622 individuals suspected for TB were identified in Sistan region and were evaluated by standard laboratory approaches like smear preparation, radiography, clinical signs, and molecular approaches. Of them, 1800 established TB patients were identified; of whom, 1523 were pulmonary TB and 277 were extra pulmonary TB. In 66 cases (3.6\%), the therapy failed and in 64 cases $(3.5 \%)$, recurrent pulmonary TB was observed.
The population of Sistan region at the start of the survey (2010) was about $455332,{ }^{10}$ hence the cumulative incidence of TB was 395 cases per 100000 during 4 years and in average, 99 cases per 100000 per year. From all the population of this region, $41 \%$ lived in urban areas and $59 \%$ lived in rural regions. During this period, 485 of TB cases $(27 \%)$ were from urban areas and 1315 cases $(73 \%)$ were from rural areas. In this research, the frequency of female and male patients was 1045 (58\%) and 755 (42\%), respectively. Table 1 shows the incidence of TB based on the type of TB, gender, and residence area per 100000 population of Sistan region.

Minimum and maximum ages of smear positive pulmonary TB cases in 2010 were 12 and 99 years old, respectively. Accordingly, the respective maximum and minimum ages were 11 and 99 years old in 2011, 14 and 97 years old in 2012, and 13 and 91 years old in 2013.

Table 2 shows age characteristics of patients in this 4 -year period. Results are presented as mean \pm standard deviation (SD).

During the study period, $65 \%$ of patients were identified by sputum smear exams, $34 \%$ of patients were identified by radiography and clinical signs, and the rest were identified by using sputum culture and antibiogram. No patient was identified by molecular techniques such as polymerase chain reaction (PCR).

In this period, 525 cases were evaluated by antibiogram performed for common antibiotics that were used as the first line of TB treatment (isoniazid, rifampin, ethambutol, and streptomycin). The tests were conducted by Masih Daneshvari hospital (Tehran, Iran). In this survey, 38 (7.3\%) drug resistant cases were identified; most of them were resistant to Isoniazid and Rifampin, and the least rate of drug resistance was related to ethambutol and streptomycin (Table 3).

\section{Discussion}

The incidence rate of TB varies in different provinces of Iran, but the highest rate was reported for Sistan and Baluchistan province. ${ }^{11}$ In the recent years, Sistan region has been called as the center of TB in Iran. ${ }^{12}$ Sistan and Balouchestan province is neighbor to Afghanistan and Pakistan and $14 \%$ of Afghan migrants live in this province. ${ }^{13}$ Afghanistan and Pakistan countries have a

Table 1. Incidence Rate of Tuberculosis in Sistan Region (2010-2013)

\begin{tabular}{|c|c|c|c|c|c|}
\hline \multirow{2}{*}{\multicolumn{2}{|c|}{ Incidence Rate Per 100000 Population }} & \multicolumn{4}{|c|}{ Year } \\
\hline & & \multirow{2}{*}{$\frac{2010}{61}$} & \multirow{2}{*}{$\begin{array}{c}2011 \\
65\end{array}$} & \multirow{2}{*}{$\begin{array}{c}2012 \\
63\end{array}$} & \multirow{2}{*}{$\begin{array}{c}2013 \\
54\end{array}$} \\
\hline \multirow{3}{*}{ Type of TB } & New pulmonary (smear positive) & & & & \\
\hline & New pulmonary (smear negative) & 18 & 17 & 18 & 11 \\
\hline & New extra pulmonary & 14 & 20 & 15 & 15 \\
\hline \multirow{2}{*}{ Residence area } & Urban & 64 & 69 & 76 & 54 \\
\hline & Rural & 126 & 131 & 129 & 110 \\
\hline \multirow{2}{*}{ Gender } & Female & 125 & 114 & 127 & 108 \\
\hline & Male & 80 & 101 & 90 & 72 \\
\hline
\end{tabular}


Table 2. Age Characteristics of Tuberculosis Patients During 2010-2013

\begin{tabular}{|c|c|c|c|c|}
\hline \multirow{2}{*}{ Age $(y)($ Mean \pm SD) } & \multicolumn{4}{|c|}{ Year } \\
\hline & 2010 & 2011 & 2012 & 2013 \\
\hline New smear positive cases & $55.5 \pm 21.8$ & $57.6 \pm 22.0$ & $56.9 \pm 20.8$ & $58.4 \pm 19.5$ \\
\hline New smear negative cases & $56.2 \pm 21.5$ & $52.7 \pm 22.0$ & $54.3 \pm 21.0$ & $53.0 \pm 20.4$ \\
\hline New extra pulmonary & $37.1 \pm 17.5$ & $44.1 \pm 22.4$ & $41.9 \pm 23.4$ & $46.3 \pm 20.3$ \\
\hline Recurrent cases & $67.4 \pm 9.8$ & $61.5 \pm 18.8$ & $67.6 \pm 13.8$ & $73.7 \pm 6.0$ \\
\hline All new cases and recurrent cases & $53.3 \pm 19.5$ & $54.4 \pm 19.8$ & $54.5 \pm 19.3$ & $55.9 \pm 17.9$ \\
\hline
\end{tabular}

Table 3. The Frequency of Drug Resistance Among Tuberculosis patients

\begin{tabular}{lc}
\hline Drug & $\begin{array}{r}\text { Resistance Rate Among Examined } \\
\text { Cultures, No. (\%) }\end{array}$ \\
\hline Isoniazid & $15(3)$ \\
Rifampin & $16(3)$ \\
Ethambutol & $0(0)$ \\
Streptomycin & $0(0)$ \\
Isoniazid + Rifampin & $7(1.3)$ \\
No resistance & $487(92.7)$ \\
Total & $525(100)$ \\
\hline
\end{tabular}

high number of TB-infected individuals. ${ }^{8}$ Afghanistan country is among the 22 countries having the highest rate of TB infection and this country is among the 6 countries having the highest rate of drug resistant TB cases. ${ }^{8} \mathrm{~A}$ survey conducted in Kashan of Iran in 1997 showed that $33 \%$ of pulmonary TB-infected individuals were Afghan migrants. ${ }^{14}$ In another survey conducted in Damghan of Iran, 22.5\% of TB-infected cases were Afghan migrants. ${ }^{15,16}$ One of the risk factors of TB has been chronic obstructive pulmonary disease (COPD). Researches have revealed that affection by COPD significantly increases the risk of TB infection and its recurrence. ${ }^{17}$ Extensive droughts in our region during 2010 and the years after that resulted in drying Hamun lake and subsequent sand storms. These storms particularly predisposed the population of Sistan to COPD. This phenomenon in turn can be partly responsible for high rate of $\mathrm{TB}$ in this region. ${ }^{18-22}$

In this research, $80 \%$ of cases were pulmonary $\mathrm{TB}$ cases. In previous studies on Iranian people, the prevalence of smear positive TB was within the range of $56.5 \%$ $77.9 \% .^{23-27}$ Smear positive TB seems as the most specific indicator for pulmonary TB. ${ }^{28}$ Our result is compatible with the previous studies, as smear positive TB was more prevalent than smear negative TB and this may refer to the importance of smear preparation as an investigation approach.

In this study, the incidence rate among women was more than that among men. In another study conducted in Qom province of Iran, $39.5 \%$ of patients were female and $60.5 \%$ of cases were male. ${ }^{23}$ In a survey conducted in Kashan city of Iran, the incidence rate among men was more than that among women. ${ }^{14}$ Moreover, in another study conducted during 1992-2001 in Kermanshah, Iran, 52.4\% of cases were male and $47.6 \%$ of patients were female. ${ }^{29}$ In another study in Ardabil of Iran, the incidence among women was more than that among men but the difference was not significant. ${ }^{28}$ In 2015 another study in Hamadan city of Iran also showed that $50.4 \%$ of cases were female. ${ }^{25}$ In another study conducted in Sistan and Balouchestan during 2005-2008, the incidence among women was more than that among men. ${ }^{11}$ In a similar survey in southeast of Iran, the incidence rates among women and men were 81.8 $\%$ and $18.2 \%$ respectively. ${ }^{30}$ The global and international reports from all around the world indicate that incidence rate of TB in males is higher than that in females at all ages except for children, in which female children developed TB more than males. The reason for male predominance is not thoroughly understood, however some studies have mentioned some explanations as difficulty in accessing health care, preference in receiving private practitioners such as traditional medicines, and lack of female sanitary centers. $^{31}$

The higher rate of incidence in females in our report can be resulted from the lifestyle of the women. In Sistan and Balouchestan province women are mostly housewives and have a lesser rate of literacy than men. They also experience successive parturitions and the impairment in body conditions and nutritional deficiency that can result in the further incidence of TB among women. ${ }^{3,23}$

In this study the frequency of the patients in rural areas was more than that in urban areas. In another study in Qom province, $90 \%$ of TB patients were from urban areas. ${ }^{23}$ Furthermore, in another study in Kashan, the majority of patients were from urban areas. ${ }^{14}$ In another study conducted in Hamadan 38\% of patients were from rural areas and $62 \%$ of them were from urban areas. ${ }^{25}$ In a study conducted by Gholami et al, it was revealed that $73 \%$ of the patients were from urban areas and $27 \%$ of them were from rural areas. ${ }^{2}$ In a survey in Arak (1996-1997), Iran, $68 \%$ of cases were from urban areas. ${ }^{32}$ In another study (2003-2007) in Damghan of Iran, 59.9\% of patients were from urban areas. ${ }^{15,23}$ However, in a study conducted by Vorokhobkin Ius et al in Russia, the incidence rate in rural area was 1.5 times greater than that in urban areas. ${ }^{33,34}$ In another study by Long et al in Canada, a higher rate of incidence was observed in urban areas. ${ }^{35}$ However in the present study, we observed the high incidence rate of this disease in rural areas that can be because of the successive droughts and the invasion of sands to rural areas and subsequent increment of risk factors of TB like COPD in 
rural areas.

In this study, the highest rate of incidence was at the ages higher than 65 while the lowest age was between 5-9 years old. In a study conducted by Saghafipour et al, 35\% of patients were 65 years of age and older. ${ }^{23}$ In the study conducted by Alaei et al, the highest rate was observed among those aged between 61 and 70 years old. ${ }^{36}$ Yet in a study in Kordestan province of Iran, the highest rate of incidence was reported for population aged within 40-70 years old. ${ }^{3}$ Moreover, in a survey conducted by Yousefnejad et al, $80 \%$ of patients were aged more than 40 and $59.5 \%$ of patients were between 40 and 70 years old. ${ }^{3}$ In a survey conducted in Hamadan, the highest age for TB patients was more than 70 years old. ${ }^{25}$ In a study conducted by Farchi et al in Italy, the highest rates were among 10-24 year-old people. ${ }^{37}$ Moreover in a study conducted by Long et al in Canada, the highest rate was observed among 65 year-old population and older. ${ }^{352}$ This trend of incidence in old ages can be because of immunosuppression and more sensitivity to pathogens in the old ages. ${ }^{38,39}$

The standardized anti-TB drugs have been used for many years and it is reasonable to expect the occurrence of any drug resistance within the patients. Multi-drug resistance (MDR) to TB is resistance to at least isoniazid and rifampin (the most powerful drugs used in the first line of TB treatment). MDR can be resulted following inappropriate treatment, insufficient use of anti-TB drugs or low quality drugs used for TB treatment. In 2014, 480000 cases of MDR TB were observed in the world, half of them were from India, Republic of China, and Russia. It is estimated that $9.7 \%$ of MDR TB cases are infected with extensively drug-resistant TB (XDR TB). ${ }^{40}$ In Iran the incidence rate of MDR-TB in cases without any history of treatment was $5 \%$, while it was $48 \%$ in previously treated cases. Therefore, it is very important to pay attention to this burden of insanitary. ${ }^{39}$ In the present study, the highest rate of resistance was related to isoniazid and rifampin while there was no resistance to streptomycin and ethambutol. In another study conducted in Azerbaijan province of Iran in 2014 the highest rate of drug resistance was to streptomycin, rifampin, and isoniazid. ${ }^{41}$

\section{Conclusion}

The present study revealed that Sistan area is an endemic region for TB; this is partially due to the climatic issues. Therefore, the people in Sistan region, especially women living in rural areas, should receive sufficient education to be protected against TB.

\section{Competing Interests}

The authors declare that they have no competing interests in this research.

\section{Ethical Approval}

We used the data collected previously by the contribution of sanitary and TB centers of Zabol city.

\section{Acknowledgments}

The authors acknowledge the ongoing friendly contributions from the Zabol TB center especially Miss Zohreh Khalili and Miss Salia Amini Pooya, students of Zabol University of Medical Sciences.

\section{References}

1. Irving WL, Ala'Aldeen DA, Boswell T. Medical Microbiology: Garland Science; 2005.

2. Gholami A, Gharehaghaji R, Moosavi-Jahromi L, Sadaghianifar A. Epidemiologic survey of pulmonary tuberculosis in Urmia city during 2004-2007. Knowledge Health. 2009:4(3):19-23.

3. Yousefnejad S MA, Mehrabi Y, Hatami H. Epidemiology of smear positive pulmonary tuberculosis in Kordestan province. Quarterly of Infections and Tropical Diseases. 2013;62:49-52.

4. Pommerville JC. Alcamo's Fundamentals of Microbiology. Sudbury, Mass: Jones Bartlett Learning; 2004.

5. Borgdorff MW, Nagelkerke NJ, de Haas PE, van Soolingen D. Transmission of Mycobacterium tuberculosis depending on the age and sex of source cases. Am J Epidemiol. 2001;154(10):934-943.

6. Murray CJ, Lopez AD. Mortality by cause for eight regions of the world: Global Burden of Disease Study. Lancet. 1997;349(9061):1269-1276. doi:10.1016/s01406736(96)07493-4

7. Nasehi ML. National Guideline for Prevention Of Tuberculosis. Tehran: Andishmand; 2010. [Persian].

8. WHO. Global tuberculosis report. Switzerland: WHO; 2015.

9. Tarverdizadeh Y, Imani Fooladi AA. Investigation of Predisposing and Environmental Factors in Tuberculosis (TB). Journal of Medical Council of Iran. 2013;30(4):335340 .

10. The estimation of the population of country cities (based on the comparison of geographic areas in 2010. https:// www.amar.org.ir/default.aspx. Accessed June 2014.

11. Meysamie A, Salehi M, Sargolzaei N. Trend of smear positive pulmonary tuberculosis in Sistan and Baluchestan Province (2005-2008). Tanaffos. 2010;9(1):48-53.

12. Sand storm crisis in sistan. Ministry of Interior Agency. https://www.moi.ir/portal/home/showpage.aspx?ob ject $=$ news \& categoryid $=541$ ac873-3706-4f31-97e75 cd6d51170eb\&webpartid $=$ cb29dce2-3092-430b-8ecf$1 \mathrm{~cd} 76588 \mathrm{f} 4 \mathrm{e} 3 \& \mathrm{id}=99 \mathrm{~d} 6 \mathrm{~b} 25 \mathrm{f}-70 \mathrm{cf}-40 \mathrm{bc}-\mathrm{b} 03 \mathrm{e}-\mathrm{fe} 968 \mathrm{~d}-$ 2bff59. Accessed May 10, 2016.

13. Migrants from stats aspect. Etemad Newspaper. October 19, 2008. http://www.magiran.com/npview.asp?ID=1724139.

14. Khalife Soltani AH, Arbabi M. Epidemiological Survey of tuberculosis in patients tuberculosis referred to Kashan 1993-1996. Feyz. 1997;4:81-88. [Persian].

15. Mohamadi Azni S, Mansourian AA, Nokandeh Z. Epidemiological study of tuberculosis in Damghan city (Iran) during 2003-2007. Koomesh. 2008;9(4):315-320. [Persian].

16. Chakrabarti B, Calverley PM, Davies PD. Tuberculosis and its incidence, special nature, and relationship with chronic obstructive pulmonary disease. Int J Chron Obstruct Pulmon Dis. 2007;2(3):263-272. 
17. Inghammar $\mathrm{M}$, Ekbom $\mathrm{A}$, Engstrom $\mathrm{G}$, et al. COPD and the risk of tuberculosis--a population-based cohort study. PLoS One. 2010;5(4):e10138. doi:10.1371/journal. pone. 0010138

18. Aktogu S, Yorgancioglu A, Cirak K, Kose T, Dereli SM. Clinical spectrum of pulmonary and pleural tuberculosis: a report of 5,480 cases. Eur Respir J. 1996;9(10):2031-2035.

19. Wang JY, Lee LN, Hsueh PR. Factors changing the manifestation of pulmonary tuberculosis. Int J Tuberc Lung Dis. 2005;9(7):777-783.

20. Liu SF, Liu JW, Lin MC. Characteristics of patients suffering from tuberculous pleuritis with pleural effusion culture positive and negative for Mycobacterium tuberculosis, and risk factors for fatality. Int J Tuberc Lung Dis. 2005;9(1):111-115.

21. Didilescu C, Ibraim E, Plopeanu D. [A study of the risk factors for relapse in pulmonary tuberculosis patients and the results of the re-treatment]. Pneumologia. 2000;49(4):247-252.

22. White MC, Portillo CJ. Tuberculosis mortality associated with AIDS and drug or alcohol abuse: analysis of multiple cause-of-death data. Public Health. 1996;110(3):185-189. doi:10.1016/S0033-3506(96)80074-6

23. Saghafipour A, Noroozei M, Mostafavi R, Heidarpour, Azam, Ghorbani, Mostafa. The epidemiologic status of Pulmonary Tuberculosis and its associated risk factors in Qom province during 2002-2010. J Mazandaran Univ Med Sci. 2012;22(90):63-70. [Persian].

24. Ahmadrajabi R AF. Epidemiology of tuberculosis in Bam (1996-2002). J hormozgan Univ Med Sci. 2002;6:1-6. [Persian].

25. Najafi Vosogh R, Roshanaei G, Khazaei S, Safari M, Zahiri A, Bothaei J. Study of Tuberculosis epidemiology and its affected factors in Hamadan province, during the years 2007-2013. Pajouhan Scientific Journal. 2015;14(1):64-71. [Persian].

26. Kadivar MR, Ghaneh-Shirazi R, Khavandegaran F, Karimi M. Epidemiology of tuberculosis among Afghan immigrants in Fars province, southern Islamic Republic of Iran. East Mediterr Health J. 2007;13(4):758-764.

27. Mohammadpour A, Fani MJ, Motalebi M, Shams H. Epidemiolgy of tuberculosis disease during 1372-80 in Gonabad city. Ofogh-E-Danesh. 2002;8(1):45-51. [Persian].

28. Hazrati S, Khaligh N, Moeini A, et al. Epidemiology of
Tuberculosis in Ardabil City from 2005 to 2010. J Health. 2013;4(2):103-109.

29. Hatami H, Haddadi P. radiographic findings of pulmonary tuberculosis patients hospitalized in Kermanshah: Sina hospital(1992-2001). Scientific Quarterly of Kermanshah University of Medical Sciences. 2005;4:1-4. [Persian].

30. Kermansaravi F, Metanat M. Prevalence of Smear Positive Pulmonary Tuberculosis among Diabetic Patients in Southwest of Iran. J Mazandaran Univ Med Sci. 2012;22(87):97-103. [Persian].

31. WHO. Gender and tuberculosis. Geneva: Dept. of Gender and Women's Health; 2002.

32. Sofian M, Zarinfar N, Mirzaee M, Moosavi-Nejad A. Epidemiology of tuberculosis in Arak, Iran. Koomesh. 2009;10(4):261-266

33. Vorokhobkin Iu S. [Age-sex-related and social characteristics of patients with new-onset tuberculosis in urban and rural areas]. Probl Tuberk Bolezn Legk. 2005(12):26-29.

34. de Vries G, van Hest NA, Baars HW, Sebek MM, Richardus $\mathrm{JH}$. Factors associated with the high tuberculosis case rate in an urban area. Int J Tuberc Lung Dis. 2010;14(7):859865.

35. Long R, NjooH,HershfieldE. Tuberculosis:3. Epidemiology of the disease in Canada. CMAJ. 1999;160(8):1185-1190.

36. Alaei K, Mansouri SD, Alaei A. Study on the prevalance rate of clinical tuberculosis in HIV positive patients in Kermanshah province 1998-2001. J Mazandaran Univ Med Sci. 2002;12(35):20-28. [Persian].

37. Farchi S, Mantovani J, Borgia P, Giorgi Rossi P. Tuberculosis incidence, hospitalisation prevalence and mortality in Lazio, Italy, 1997-2003. Int J Tuberc Lung Dis. 2008;12(2):193-198.

38. WHO. Tubetculosis control in the south east Asia Region. Geneva, Switzerland: WHO; 2016.

39. WHO. Tuberculosis country profiles: Iran tuberculosis profile. http://www.who.int/tb/country/data/profiles/en/. Accessed May 4, 2016.

40. WHO. Tuberculosis: fact sheet. http://www.who.int/ mediacentre/factsheets/fs104/en/. Accessed April 2016.

41. Ansarin K, Sahebi L, Khalili M, et al. Epidemiology and Patterns of Drug Resistance in Pulmonary Tuberculosis in East Azerbayjan. Med J Tabriz Univ Med Sci Health Services. 2015;36(6):6-13. [Persian]. 